\title{
In vitro fertilizing potential of urethral and epididymal spermatozoa collected from domestic cats (Felis catus)
}

\author{
S. Prochowska, W. Niżański \\ Wrocław University of Environmental and Life Sciences, Faculty of Veterinary Medicine, \\ Department of Reproduction and Clinic of Farm Animals, \\ pl. Grunwaldzki 49, 50-366 Wrocław, Poland
}

\begin{abstract}
The aim of this study was to provide a comparative analysis of in vitro fertilizing potential of frozen-thawed urethral and epididymal feline spermatozoa. Both types of semen were collected from 7 cats and cryopreserved in liquid nitrogen. To perform in vitro fertilization, both urethral and epididymal samples from the same individual were thawed and spermatozoa were co-incubated with in vitro matured cat oocytes. Obtained embryos were cultured in vitro for 7 days in a commercial medium. Cleavage rate, morula rate and blastocyst rate were calculated. Experiment was run in 10 replicates. The examined parameters showed no significant differences between urethral and epididymal spermatozoa $(p>0.05)$. Cleavage rate and embryo's development were highly variable between replicates, even for the different sperm samples collected from one individual. There was no significant correlation between fertilizing capacity of two types of spermatozoa collected from the same male. In this study we confirmed that cryopreserved urethral spermatozoa have equally good fertilizing potential as epididymal ones, and both can be successfully used for in vitro fertilization in cats with the use of commercial medium.
\end{abstract}

Key words: cat spermatozoa, urethral semen, epididymal semen, sperm fertilizing potential, in vitro fertilization

\section{Introduction}

From 36 species of wild felids, all of them are classified as endangered in at least part of its area of distribution (Pope 2000). During past decades scientists put a lot of effort to implement Assisted Reproductive Techniques (ART) into rescue programs of endangered felines, with the development of such techniques as cryopreservation of gametes, artificial insemination, in vitro fertilization, ICSI and embryo transfer. As studies on rare species are difficult to conduct, the domestic cat serves as a model animal (Pope 2000). One of the factors limiting the use of ART in felids in the past was difficulty in obtaining spermatozoa from tomcats. Collection of urethral semen proposed by Zambelli et al. (2008) seems to solve 
this problem, but the characteristics of spermatozoa collected by this method are still poorly studied. Especially its fertilizing ability is not well understood, with only two papers concerning this subject (Zambelli et al. 2008, Filliers et al. 2010).

For in vitro fertilization in cats several studies were conducted, with the use of different types of semen, miscellaneous media and various culture conditions (Freistedt et al. 2001, Herric et al. 2007, Waurich et al. 2010, Thuwanut et al. 2015). The results of these studies show a large variation and it is hard to compare fertilizing potential of spermatozoa obtained by different methods. For urethral and epididymal spermatozoa a direct comparison was made only for fresh samples (Filliers et al. 2010), the data for frozen-thawed spermatozoa are lacking. Cryopreserved semen is more useful for ART, although it is generally acknowledged that fertility obtained with frozen semen is lower than with fresh, for multiple reasons (Watson 2000). Therefore fertilizing potential of frozen-thawed spermatozoa needs to be further investigated.

The aim of this study was to compare in vitro fertilizing potential of cryopreserved urethral and epididymal spermatozoa collected from domestic cats.

\section{Materials and Methods}

\section{Media}

All laboratory chemicals and reagents used in this study were purchased from Sigma-Aldrich Poland, until stated otherwise. Tris buffer used as a basic semen extender contained $3.02 \%(\mathrm{w} / \mathrm{v})$ Tris, $1.35 \%$ $(\mathrm{w} / \mathrm{v})$ citric acid, $1.25 \%(\mathrm{w} / \mathrm{v})$ fructose, in bidistilled water; $\mathrm{pH}$ 6.5. Freezing extender contained Tris buffer supplemented with $20 \%(\mathrm{v} / \mathrm{v})$ egg yolk, $6 \%$ glycerol (v/v) and 1\% Equex STM paste (v/v) (Nova Chemical Sales Inc., Scituate, MA, USA). Washing medium (WM) used for collection and rinsing of oocytes consisted of Medium 199 containing Earle's salts, supplemented with $3 \mathrm{mg} / \mathrm{ml} \mathrm{BSA,} 0.1 \mathrm{mg} / \mathrm{ml}$ cysteine, 1.4 $\mathrm{mg} / \mathrm{ml}$ HEPES, $0.25 \mathrm{mg} / \mathrm{ml}$ sodium pyruvate, 0.6 $\mathrm{mg} / \mathrm{ml}$ sodium lactate, $0.15 \mathrm{mg} / \mathrm{ml} \mathrm{L}$-glutamine, and $0.055 \mathrm{mg} / \mathrm{ml}$ gentamicin (Waurich 2010). Maturation medium (MM) for in vitro maturation of oocytes consisted of WM supplemented with $0.02 \mathrm{IU} / \mathrm{ml}$ of FSH and $0.02 \mathrm{IU} / \mathrm{ml} \mathrm{LH}$. For in vitro fertilization and embryoculture we used commercial media purchased from Irvine Scientific (Irvine Scientifc, Santa Ana, CA, USA): Human Tubular Fluid (HTF) and Continuous Single Culture medium (CSC), both substituted with $10 \%$ of Serum Substitute Suplement (SSS).
Animals

Urethral semen, testes and ovaries were obtained from the Domestic Shorthair cats intended for routine castration procedures at the Department of Reproduction and Clinic of Farm Animals of Wroclaw University of Environmental and Life Sciences (Poland). The ovaries were kept in phosphate-buffered saline with penicillin G sodium 1,000,000 I.U./ml, streptomycin sulphate $100 \mathrm{mg} / \mathrm{ml}$ and amphotericin B $250 \mu \mathrm{g} / \mathrm{ml}$ (Luvoni et al. 2006) at $4^{\circ} \mathrm{C}$ up to $24 \mathrm{~h}$ before processing, whereas the testes were processed on the same day. All procedures were performed with the consent of the Second Local Ethical Committee in Wroclaw.

\section{Semen collection and assessment}

Semen was collected between April and October from 7 mix-breed tomcats (aged from 8 months to 6 years). From each tomcat urethral semen (CT) was collected by urethral catheterization after medetomidine administration (medetomidine hydrochloride im $100 \mu \mathrm{g} / 1 \mathrm{~kg}$ of body weight (Sedator 1.0 $\mathrm{mg} / \mathrm{ml}$, Novartis, Poland)) according to Zambelli et al. (2008). Immediately after collection of urethral semen, ketamine im $5 \mathrm{mg} / 1 \mathrm{~kg}$ of body weight (VetKetam $100 \mathrm{mg} / \mathrm{ml}$, VetAgro, Poland) was administered and orchiectomy performed. Within 5-10 min after the removal of the testes, epididymal spermatozoa (EP) were collected by epididymal slicing (Niżański et al. 2005). In every sample sperm motility was assessed subjectively under a contrast phase microscope (magnification x200) and sperm concentration was calculated by the Thoma chamber.

\section{Semen cryopreservation and thawing}

Semen cryopreservation was performed using the same protocol as previously described (Niżański et al. 2005). After centrifugation at $620 \mathrm{xg}$ for $5 \mathrm{~min}$, the supernatant was removed and the sperm pellet was re-suspended at room temperature in freezing extender. After re-suspension, sperm samples were cooled to $5^{\circ} \mathrm{C}$ within $1 \mathrm{~h}$, equilibrated at $5^{\circ} \mathrm{C}$ for $1.5 \mathrm{~h}$, loaded into pre-cooled $0.25 \mathrm{ml}$ straws cut to made $0.125 \mathrm{ml}$ straw $\left(5 \times 10^{6} / \mathrm{ml}\right.$ spermatozoa per straw) and held $5 \mathrm{~cm}$ above the surface of liquid nitrogen for $10 \mathrm{~min}$. The straws were kept at $-196^{\circ} \mathrm{C}$ until the use for in vitro fertilization. For each replicate $(n=10)$, cryopreserved CT and EP samples collected from the same male were thawed by immersion in a $37^{\circ} \mathrm{C}$ water bath for $30 \mathrm{~s}$. Each sample was centrifuged at $620 \mathrm{xg}$ for $5 \mathrm{~min}$, supernatant was discarded and the pellet were re-sus- 
Table 1. The results of in vitro fertilization and embryo culture with the use of frozen-thawed urethral (CT) and frozen-thawed epididymal (EP) cat spermatozoa. Number of replicates: $n=10$.

\begin{tabular}{ccccccc}
\hline & \multicolumn{2}{c}{ Oocytes } & & Morula rate & Blastocyst rate \\
\cline { 2 - 4 } & total & matured & & Cleavage rate & & \\
\hline CT & 118 & 64 & & $51.6 \%(33 / 64)^{\mathrm{a}}$ & $60.6 \%(20 / 33)^{\mathrm{a}}$ & $30.3 \%(10 / 33)^{\mathrm{a}}$ \\
\hline $\mathrm{EP}$ & 113 & 67 & $53.7 \%(36 / 67)^{\mathrm{a}}$ & $41.7 \%(15 / 36)^{\mathrm{a}}$ & $19.4 \%(7 / 36)^{\mathrm{a}}$ \\
\hline
\end{tabular}

$\mathrm{a}, \mathrm{b}$ - different superscripts within columns indicate significant differences $\mathrm{p}<0.05$.

Cleavage rate - percentage of cleaved embryos assessed at Day 1 relative to the number of matured oocytes; morula rate - percentage of embryos developed to at least morula stage at Day 6 relative to the number of cleaved embryos; blastocyst rate - percentage of embryos developed to blastocyst stage at Day 7 relative to the number of cleaved embryos.

pended in HTF+SSS. The concentration of motile spermatozoa was adjusted to $4 \times 10^{6} / \mathrm{ml}$ (Waurich et al. 2010).

\section{In vitro fertilization and embryo culture}

Oocytes with surrounding cumulus (COC) were collected by slicing the ovarian cortex with scalpel blade in WM. COCs possessing dark, uniform cytoplasm and surrounded by at least several layers of cumulus cells were subjected to in vitro maturation (IVM) in $\mathrm{MM}$ at $38.5^{\circ} \mathrm{C}$ in $5 \% \mathrm{CO}_{2}$, for 24 $\mathrm{h}$ (Waurich et al. 2010). The in vitro matured oocytes were washed twice in WM, randomly divided into two groups (9-11 oocytes per group) and transferred to the separate wells containing $300 \mu \mathrm{l}$ of $\mathrm{HTF}+\mathrm{SSS}$. A hundred microliters of $\mathrm{CT}$ was added to one well and $100 \mu \mathrm{l}$ of EP to the second. Co-incubation of gametes lasted for $18 \mathrm{~h}$ at $38.5^{\circ} \mathrm{C}$ in $5 \% \mathrm{CO}_{2}$ (Waurich et al. 2010). Next, the presumptive zygotes were washed 2 times in $\mathrm{WM}$ and moved to the well containing $400 \mu \mathrm{l}$ of CSC+SSS. The cleavage rate (the percentage of embryos with the presence of at least two decent blastomeres) was determined $24 \mathrm{~h}$ from the start of IVF. The embryos were transferred to the individual drops of $30 \mu \mathrm{l}$ of CSC+SSS and cultured in vitro for 7 days. The embryo development was assessed daily and embryos with obvious degenerative changes or arrested were removed from the culture. At day 5 embryos were transferred to fresh drops of CSC+SSS. At day 6 the morula rate (the percentage of embryos developed to at least morula stage relative to the number of cleaved embryos) and at the day 7 blastocyst rate (the percentage of embryos developed to blastocyst stage relative to the number of cleaved embryos) were calculated. The experiment was conducted in 10 replicates. Semen of one individual was used in 4 replicates, in other 6 replicates semen from other 6 males were used once per replicate.

\section{Data analysis}

Data obtained for in vitro fertilization and embryo culture were analyzed with Chi-square test. The comparison of subjective motility of two types of spermatozoa was conducted with student's t-test. The Pearson correlation was calculated to compare the in vitro fertilization results for CT and EP from the same individual. The level of significance was set at $\mathrm{p}<0.05$. An elaboration of tests was carried out using the Statistica software for Windows, StatSoft Polska Sp. z o.o.

\section{Results}

Both types of spermatozoa showed comparable quality ( $>0.05$ ), with an average subjective motility in CT $73.8 \pm 18.5 \%$ and in EP $64.4 \% \pm 5.2$. After cryopreservation subjective motility was $43.5 \pm 12.5 \%$ in $\mathrm{CT}$ and $39.5 \pm 9.0 \%$ in EP, and no significant difference between two types of spermatozoa was observed $(\mathrm{p}>0.05)$.

In our study we achieved similar percentage of early stage embryos (cleavage rate) in both CT and EP group (Table 1). Although we observed a tendency for better development of embryos to the morula or blastocyst stage for CT spermatozoa, the difference was not significant (Table 1). Large variations among individual embryocultures were observed, regardless of the type of semen used for in vitro fertilization - in some cases none of the embryos reached the blastocyst stage $(0 \%)$, in others the majority of them (up to $75 \%$ ). There were no significant correlations between CT and EP sperm samples collected from the same male (Fig. 1), especially the percentage of blastocysts seemed not to be related (Fig. 1C). Also, when the semen from the same individual was used in different replicates, the results were highly variable (for CT cleavage rate ranged from $33 \%$ to $63 \%$, morula rate from $0 \%$ to 

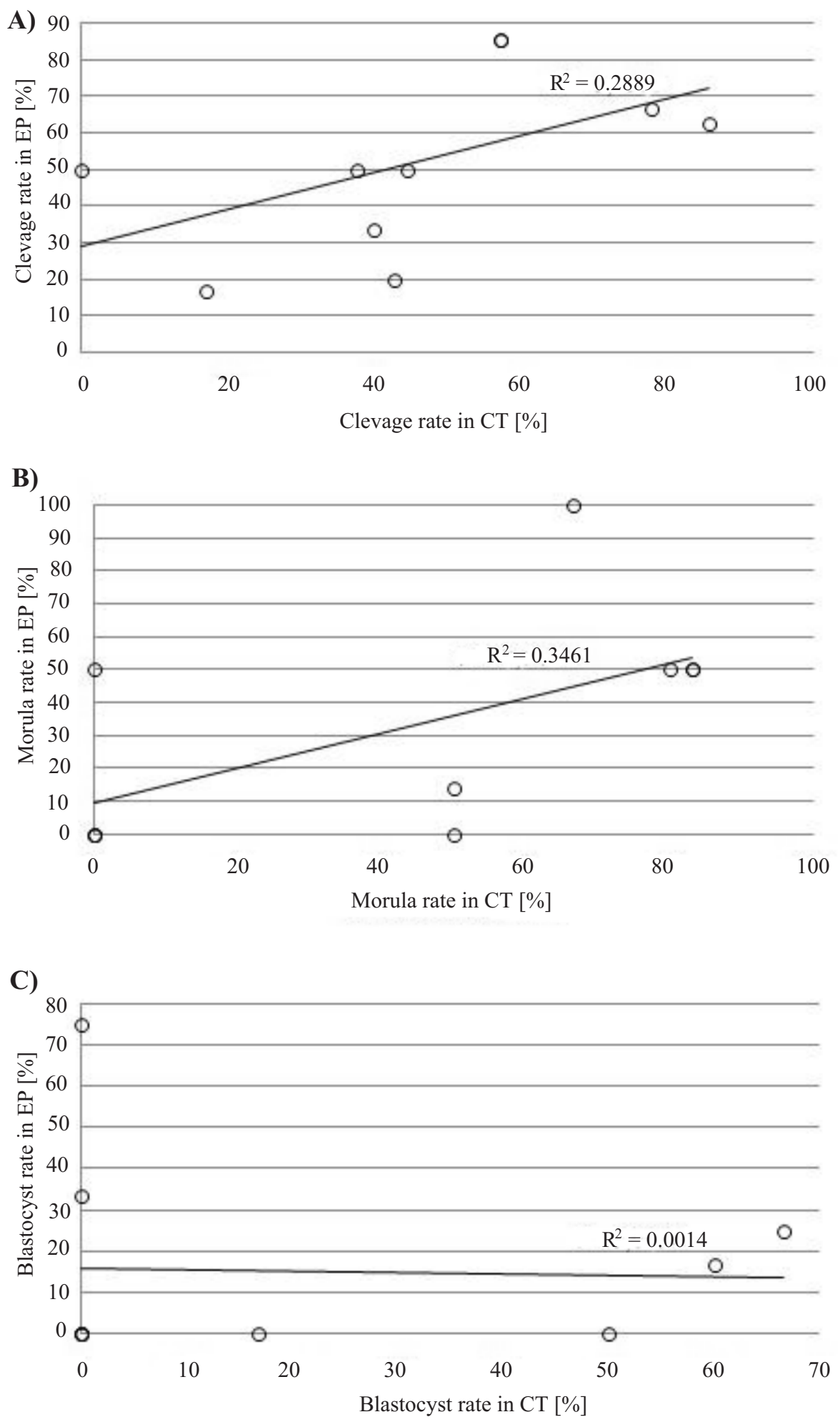

Fig. 1. Cleavage rate (A), morula rate (B) and blastocyst rate (C) for frozen-thawed urethral (CT) and frozen-thawed epididymal (EP) cat spermatozoa collected from the same individual. Number of replicates: $n=10$. 
$80 \%$ and blastocyst rate from $0 \%$ to $60 \%$; for EP cleavage rate was between $40 \%$ and $86 \%$, morula rate varied from $0 \%$ to $50 \%$ and blastocyst rate from $0 \%$ to $17 \%$ ).

\section{Discussion}

This study was the first to compare in vitro fertilizing capacity of cryopreserved urethral and epididymal spermatozoa as paired observations. Because no significant differences were found, we can state that spermatozoa from both types of semen equally retain their ability to produce embryos after freezing-thawing. The present study completes our previous reports about comparable characteristics of spermatozoa collected from the urethra and epididymis (Prochowska et al. 2015, Prochowska et al. 2016) and finishes an overall picture of the quality of urethral semen.

Our findings are in agreement with the study of Zambelli et al. (2008), in which no significant differences in the ability of in vitro production of embryos were found between cryopreserved urethral and electroejaculated spermatozoa. Our results are also supplementary to those of Filliers et al. (2010), who showed that fertilizing ability was not different when fresh urethral and epididymal spermatozoa were used. For other species, the papers concerning this subject are scarce. In mice, cryopreserved epididymal spermatozoa showed worse ability to penetrate cumulus cells than ejaculated, which was accompanied by worse motility characteristics and a different capacitation pattern ( $\mathrm{Li}$ et al. 2015). On the contrary, in swine cryopreserved epididymal semen appeared to be superior to ejaculated, both fresh and frozen-thawed, and the authors linked it with high post-thaw motility of epididymal spermatozoa (Rath and Niemman 1997). For cats, we did not observe differences in the post thawed motility, viability, acrosome integrity or mitochondrial function between urethral and epididymal sperm cells (Prochowska et al. 2016), which may explain lack of difference in fertilizing potential. This study seems to prove that both types of spermatozoa comparably maintain anatomical and functional features that are necessary to penetrate and fertilize an oocyte.

For cats, several studies were conducted to optimize the content of media for in vitro fertilization and further embryo culture (Bogliolo et al. 2001, Herric et al. 2007, Sananmuang et al. 2011). However, preparation of complex media is often workand time-consuming. The simplification of this process may contribute to the wider use of this tech- nique in cats. Also, potential errors during the preparation of media may lower the success of in vitro fertilization. It was shown for mice that differences in the concentration of the components of media alter in vitro development of mice embryos (Lawitts and Biggers 1991). In this study we used commercial media designed for human in vitro embryoculture. Our results are comparable with these of other authors, who usually achieve the cleavage rate $>40 \%$, and the morula and blastocyst rate between 20-60\% (Freistedt et al. 2001, Zambelli et al. 2008, Thuwanut et al. 2015). Therefore, we can conclude that the commercial human media used in our study occurred to be suitable for in vitro fertilization in cats.

The development and survival of embryos in this study was highly variable, regardless of the type of semen used for in vitro fertilization. In other species, differences between individuals and variations among ejaculates from the same male affected the outcomes of in vitro fertilization (Rath and Niemman 1997, Zhang et al. 1997). Therefore, the influence of the individual tomcat (which fertility was not known) may be an explanation for the observed deviation of embryonic development. However, when we used the sperm sample from one male for subsequent replicates, the outcome also was highly variable, which do not support this hypothesis. The quality of oocytes is considered to be one of the variables influencing the outcome of in vitro fertilization (Zambelli et al. 2006). Although we tried to avoid this by choosing only the best quality oocytes (I and II grade, according to Zambeli et al. 2006), it seems that in our experiment oocytes were the only source of variability (the same procedure, the same media, the same person manipulated the oocytes). Therefore, it is probable that the developmental competence of an individual oocyte might have influenced our results. The causes of variation among the development of embryos need to be investigated further.

\section{Conclusion}

In our study we proved that both cryopreserved urethral and epididymal spermatozoa are equally good in terms of in vitro fertilizing potential and further embryo development. These findings are of great importance for further development of ART in felids, as collecting urethral semen is very convenient to use in clinical practice, and epididymal semen is still an invaluable source of spermatozoa possible to obtain after castration or death of the animal. 


\section{Acknowledgements}

The study was financed with a Polish Science Ministry grant: MNiSW/NCN N N308 576540. The authors are grateful to M. Sc. Barbara Smalec for her excellent technical assistance.

\section{References}

Bogliolo L, Leoni G, Ledda S, Naitana S, Zedda M, Carluccio A, Pau S (2001) Intracytoplasmic sperm injection of in vitro matured oocytes of domestic cats with frozen-thawed epididymal spermatozoa. Theriogenology 56: 955-967.

Filliers M, Rijsselaere T, Bossaert P, Zambelli D, Anastasi P, Hoogewijs M, Van Soom A (2010) In vitro evaluation of fresh sperm quality in tomcats: a comparison of two collection techniques. Theriogenology 74: 31-39.

Freistedt P, Stojkovic M, Wolf E (2001) Efficient in vitro production of cat embryos in modified synthetic oviduct fluid medium: effects of season and ovarian status. Biol Reprod 65: 9-13.

Herrick JR, Bond JB, Magarey GM, Bateman HL, Krisher RL, Dunford SA, Swanson WF (2007) Toward a feline-optimized culture medium: impact of ions, carbohydrates, essential amino acids, vitamins, and serum on development and metabolism of in vitro fertilization-derived feline embryos relative to embryos grown in vivo. Biol Reprod 76: 858-870.

Lawitts JA, Biggers JD (1991) Overcoming the 2-cell block by modifying standard components in a mouse embryo culture medium. Biol Reprod 45: 245-251.

Li H, Hung PH, Suarez SS (2015) Ejaculated mouse sperm enter cumulus-oocyte complexes more efficiently in vitro than epididymal sperm. PLoS One 10: e0127753.

Luvoni GC, Chigioni S, Perego L, Lodde V, Modina S, Luciano AM (2006) Effect of gonadotropins during in vitro maturation of feline oocytes on oocyte-cumulus cells functional coupling and intracellular concentration of glutathione. Anim Reprod Sci 96: 66-78.

Niżanski W, Dejneka GJ, Klimowicz M, Dubiel A (2005) Evaluating some properties of domestic cat epididymal spermatozoa and their cryopreservation. Med Weter 61: 173-178.
Pope CE (2000) Embryo technology in conservation efforts for endangered felids. Theriogenology 53: 163-174.

Prochowska S, Niżański W, Ochota M, Partyka A (2015) Characteristics of urethral and epididymal semen collected from domestic cats - A retrospective study of 214 cases. Theriogenology 84: 1565-1571.

Prochowska S, Niżański W, Partyka A. (2016) Comparative analysis of in vitro characteristics of fresh and frozen-thawed urethral and epididymal spermatozoa from cats (Felis domesticus). Theriogenology 86: 2063-2072.

Rath D, Niemann H (1997) In vitro fertilization of porcine oocytes with fresh and frozen-thawed ejaculated or frozen-thawed epididymal semen obtained from identical boars. Theriogenology 47: 785-793.

Sananmuang T, Tharasanit T, Nguyen C, Phutikanit N, Techakumphu M (2011) Culture medium and embryo density influence on developmental competence and gene expression of cat embryos. Theriogenology 75: 1708-1719.

Thuwanut P, Arya N, Comizzoli P, Chatdarong K (2015) Effect of extracellular adenosine 5'-triphosphate on cryopreserved epididymal cat sperm intracellular ATP concentration, sperm quality, and in vitro fertilizing ability. Theriogenology 84: 702-709.

Watson PF (2000) The causes of reduced fertility with cryopreserved semen. Anim Reprod Sci 60-61: 481-492.

Waurich R, Ringleb J, Braun BC, Jewgenow K (2010) Embryonic gene activation in in vitro produced embryos of the domestic cat (Felis catus). Reproduction 140: $531-540$.

Zambelli D, Merlo B, Iacono E, Prati F, Belluzzi S (2006) Fertilizing ability of electro-ejaculated cryopreserved semen in the domestic cat. Reprod Domest Anim 41: $137-141$.

Zambelli D, Prati F, Cunto M, Iacono E, Merlo B (2008) Quality and in vitro fertilizing ability of cryopreserved cat spermatozoa obtained by urethral catheterization after medetomidine administration. Theriogenology 69: 485-490.

Zhang BR, Larsson B, Lundeheim N, Rodriguez-Martinez $\mathrm{H}$ (1997) Relationship between embryo development in vitro and 56-day nonreturn rates of cows inseminated with frozen-thawed semen from dairy bulls. Theriogenology 48: 221-231. 